\title{
Exploratory investigation of the HIPPO gas-jet target fluid dynamic properties
}

\author{
Zach Meisel $^{\mathrm{a}, *}, \mathrm{Ke} \mathrm{Shi}^{\mathrm{b}}$, Aleksandar Jemcov $^{\mathrm{b}}$, Manoel Couder $^{\mathrm{a}}$ \\ ${ }^{a}$ Department of Physics, Joint Institute for Nuclear Astrophysics, University of Notre Dame, Notre Dame, IN 46556, USA \\ ${ }^{b}$ Hessert Laboratory for Aerospace Research, Department of Aerospace and Mechanical Engineering, University of Notre Dame, Notre \\ Dame, IN 46556, USA
}

\begin{abstract}
In order to optimize the performance of gas-jet targets for future nuclear reaction measurements, a detailed understanding of the dependence of the gas-jet properties on experiment design parameters is required. Common methods of gas-jet characterization rely on measuring the effective thickness using nuclear elastic scattering and energy loss techniques; however, these tests are time intensive and limit the range of design modifications which can be explored to improve the properties of the jet as a nuclear reaction target. Thus, a more rapid jet-characterization method is desired. We performed the first steps towards characterizing the gas-jet density distribution of the HIPPO gas-jet target at the University of Notre Dame's Nuclear Science Laboratory by reproducing results from ${ }^{20} \mathrm{Ne}(\alpha, \alpha){ }^{20} \mathrm{Ne}$ elastic scattering measurements with computational fluid dynamics (CFD) simulations performed with the state-of-the-art CFD software ANSYS Fluent. We find a strong sensitivity to experimental design parameters of the gas-jet target, such as the jet nozzle geometry and ambient pressure of the target chamber. We argue that improved predictive power will require moving to three-dimensional simulations and additional benchmarking with experimental data.
\end{abstract}

\section{Keywords:}

gas-jet target; ANSYS Fluent; computational fluid dynamics; recoil mass separator

PACS: 29.25.Pj, 51.30.+i

\section{Introduction}

Precise $(\alpha, \gamma)$ reaction cross sections are crucial to predict the nucleosynthesis and nuclear energy generation that occurs in a variety of stellar and explosive helium-burning environments $[1,2]$. Owing to the relatively low energies of nuclei in the relevant astrophysical conditions, traditional nuclear reaction measurements in which an outgoing $\gamma$-ray is measured often suffer from prohibitively high $\gamma$-backgrounds [3]. One approach which has been adopted to overcome this difficulty employs the so-called recoil separator, in which the nuclear recoil produced in the $(\alpha, \gamma)$ reaction is electromagnetically separated from unreacted beam nuclei and identified with a combination of time-offlight and energy-loss measurements [4].

The recoil separator technique generally relies on using inverse kinematics, where a heavy ion beam is impinged on a lighter nuclear target. As such, $(\alpha, \gamma)$ reactions require a gaseous helium target to be employed. In order to more easily capture the recoil nuclei, which are emitted from the target location with some angular distribution, recoil separators benefit from having a small target region [4]. To this end, the HIPPO gas-jet target [5] was developed to serve as the helium target for the St. George recoil sep-

\footnotetext{
* Corresponding author

Email address: zmeisel@nd.edu (Zach Meisel)
}

arator [6] at the University Notre Dame's Nuclear Science Laboratory (NSL).

In order to be suitable as a target for nuclear reaction studies with St. George, HIPPO must have a confined (relative to the size of the ion-beam) region of gaseous helium where the density is as high and uniform as possible. To achieve these properties, HIPPO produces a supersonic gas-jet in a windowless, differentially pumped volume. Though the properties of the gas-jet that is produced by HIPPO are reported in Reference [5], the measurement techniques used provide indirect information about the gas-jet volumetric density distribution and are unable to map small-scale properties of the gas-jet. The ambiguity of the inferred volumetric density distribution has the potential to introduce systematic uncertainties in the absolute cross section determined by nuclear reaction measurements performed with St. George. Additionally, considerable experimental effort is required to explore even a small number of design modifications in an effort to improve HIPPO's suitability as a nuclear reaction target for St. George. Therefore, a more rapid method of exploring design modifications is desired.

In order to investigate the properties of the HIPPO gasjet target, we have performed computational fluid dynamics (CFD) simulations with the software ANSYS Fluent. We have explored the sensitivity of the gas-jet density distribution to variations in HIPPO design parameters and 
compared our results to experimental data. Our exploratory work paves the way for future CFD simulations and experimental work which will eventually enable the rapid exploration of modifications to the design of HIPPO and future gas-jets to improve their suitability as nuclear reaction targets.

We discuss the gas-jet thickness measurements which we compare to our simulation results in Section 2, present our CFD simulations in Section 3, and the method to process our simulation results to compare to experimental data in Section 4. We discuss our results in Section 5 and conclude with closing remarks in Section 6 .

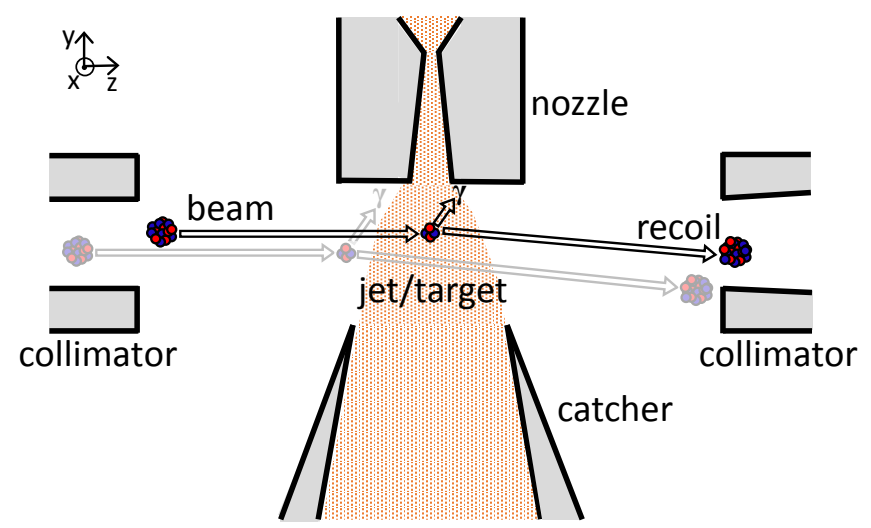

Figure 1: (color online.) Schematic of the HIPPO gas-jet target, viewed perpendicular to the ion beam and gas-jet axes. The opaque (translucent) nuclear reaction sequence demonstrates that reactions with the maximum expected recoil angle occurring within (outside of) the jet-target region are accepted into (rejected from) the St. George recoil separator.

\section{Gas-jet thickness measurements}

HIPPO is a windowless, supersonic gas-jet contained with differential pumping that employs recirculating gas flow [5]. The gas-jet, which has an aerial density of $\gtrsim$ $10^{17}$ atoms $/ \mathrm{cm}^{2}$ and a spatial extent of $\sim 3 \times 8 \mathrm{~mm}^{2}$ as seen by the ion beam, serves as the nuclear reaction target for the St. George recoil separator [6] at the NSL, as shown schematically in Figure 1. The jet is produced by flowing pure gas compressed to a pressure of at least one bar through an axisymmetric convergent-divergent nozzle into a chamber with an ambient pressure of a few millibar, where the underexpanded gas-jet is captured by a gascatcher after free-streaming for a distance of $8 \mathrm{~mm}$. The central chamber of HIPPO, which contains the gas-jet, is separated from beam-line vacuum pressures of $\sim 10^{-9}$ bar by separately pumped chambers that are connected along the beam axis by narrow apertures that accommodate the incoming and outgoing nuclei, but provide large gasflow impedances. During reaction measurements, the central chamber is generally surrounded by a close-packed $\gamma$ detection array to detect $\gamma$-rays emitted by the reaction,
Table 1: Relative maximum elastic scattering yield (in arbitrary units) and full-width at half-maximum (FWHM) from a Gaussian fit to yield profiles measured [5] for vertical $(y)$ deflections toward $(+)$ and away-from (-) the gas-jet nozzle. A vertical deflection of $0 \mathrm{~mm}$ corresponds to the mid-point between the nozzle and catcher, i.e. $4 \mathrm{~mm}$ from the nozzle and $4 \mathrm{~mm}$ from the catcher. In all cases the maxima are located along the nozzle-catcher axis, the yield uncertainty is \pm 0.50 , and the FWHM uncertainty is $\pm 0.20 \mathrm{~mm}$.

\begin{tabular}{ccc}
\hline$y$-deflection $[\mathrm{mm}]$ & Max. Yield [a.u.] & FWHM $[\mathrm{mm}]$ \\
\hline+1.78 & 16.0 & 1.85 \\
+0.89 & 14.3 & 1.90 \\
0 & 12.0 & 2.15 \\
-0.89 & 11.0 & 2.25 \\
+1.78 & 9.8 & 2.30
\end{tabular}

while a collimated silicon detector fixed at a forward angle is used to monitor the incoming ion-beam current. A full description of the HIPPO gas-jet target and target thickness measurements is provided in Reference [5].

The HIPPO gas-jet thickness measurement presented in Reference [5] employed a $2 \mathrm{MeV}^{20} \mathrm{Ne}$ beam, produced by the KN Van de Graaff accelerator at the NSL, impinging on a 1 bar (at the nozzle inlet) helium gas-jet produced by HIPPO. A collimated silicon detector, located at the end of an extension from the central chamber fixed at a forward angle (See in Figure 8 of Reference [5].), was used to measure elastically-scattered $\alpha$-particles from ${ }^{20} \mathrm{Ne}(\alpha, \alpha){ }^{20} \mathrm{Ne}$. Normalization of the scattering yield was provided by a Faraday cup following the gas-jet which measured the incoming ${ }^{20} \mathrm{Ne}$ beam current. A pair of horizontal and vertical electrostatic steerer plates located in the beam-line prior to HIPPO were used to scan the ion-beam over different locations on the gas-jet, where collimators before and after the steerers were used to confine the beam to a diameter smaller than the gas-jet.

The number of elastic scattering events detected $N_{\text {det }}$ is the product of the number of beam particles impinging the jet over some time $N_{\mathrm{p}}$, the aerial number density of the jet target atoms in units of atoms $/ \mathrm{cm}^{2} N_{\mathrm{t}}$, the probability of a beam and jet particle scattering a jet particle at a given angle $\frac{d \sigma}{d \Omega}(\theta)$, the solid angle covered by the detection system $d \Omega$, detection efficiency for the scattered $\alpha$-particle $\epsilon$, and the fraction of the time in which the data acquisition system was not busy (i.e. 'live') and could acquire new data $f_{\text {live }}{ }^{1}$ :

$$
N_{\text {det }}=N_{\mathrm{p}} N_{\mathrm{t}} \frac{d \sigma}{d \Omega}(\theta) d \Omega \epsilon f_{\text {live }} .
$$

For the $\alpha$-particles at the measured energies $\epsilon=1$ for the employed silicon detector, $f_{\text {live }}$ was reported by the acqui-

\footnotetext{
${ }^{1}$ An additional correction factor $F$ was included in the form of this equation presented in Reference [7] to account for the fact that their detection set-up would not see all elastically scattered recoils from their jet.
} 
sition system when data was taken, measurements with radioactive sources and calipers determined $\theta=64.8^{\circ} \pm 0.3^{\circ}$ and $\Omega=10.0 \pm 1.4 \times 10^{-6}$ steradians, and $\frac{d \sigma}{d \Omega}(\theta)$ was evaluated assuming Rutherford scattering. The Faraday cup located downstream from the gas-jet was used to determine the number of beam particles which impinged upon the gas-jet $N_{\mathrm{p}}$, where the measured average charge state $(q=2.3)$ of unreacted projectiles exiting the jet was used to convert between the Faraday cup current and the incoming beam intensity.

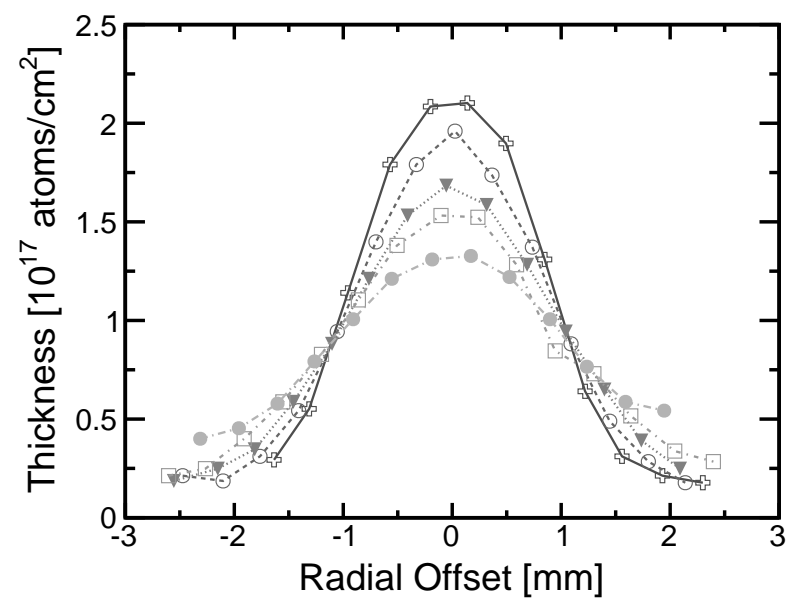

Figure 2: Target thickness inferred from the elastic scattering yield from Reference [5] for a 1 bar (inlet pressure) helium jet from HIPPO using their optimum nozzle-catcher configuration for a $2 \mathrm{MeV}^{20} \mathrm{Ne}$ beam impinging on the jet at various radial and axial positions. The five sets of data, in order of decreasing peak thickness, correspond to axial distances from the nozzle of $2.22 \mathrm{~mm}, 3.11 \mathrm{~mm}, 4.0 \mathrm{~mm}$, $4.89 \mathrm{~mm}$, and $5.89 \mathrm{~mm}$. Note that Figure 9 of Reference [5], which presents this data, has a typographical mistake in the legend, accidentally swapping the $2.22 \mathrm{~mm}$ and $3.11 \mathrm{~mm}$ cases ("0.89 $\mathrm{mm} \mathrm{Up}$ " and "1.78 mm Up" in Reference [5], respectively).

The ratio of the number of detected scattering events to the number of incoming projectiles is the yield $Y$. It is apparent that, since all other quantities are known and fixed for the duration of the elastic scattering measurement,

$$
Y \propto N_{\mathrm{t}} .
$$

Therefore, by measuring the elastic scattering yield $Y$ when scanning the incoming beam over the spatial extent of the gas-jet, one measures the spatial distribution of the aerial atomic number density $N_{\mathrm{t}}$. For a helium jet with a pressure of 1 bar (for their final nozzle-catcher geometry), Reference [5] reports that their scattering yield $Y=12 \pm 0.5$ corresponds to $N_{\mathrm{t}}=(1.68 \pm 0.14) \times 10^{17}$ atoms $/ \mathrm{cm}^{2}$. We use this relationship between the yield and the aerial number density (which is reported for the 'non-deflected' beam, which impinged the jet $4 \mathrm{~mm}$ from the nozzle, centrally along the nozzle-catcher axis) to convert reported [5] yields to aerial number densities. Yields at various locations along the jet were obtained by adjusting the voltage of electrostatic steerer plates, where the deflection angle (and therefore beam-impact position at the jet) was determined by the steerer plate geometry. The yield was measured for horizontal (' $x$ ') and vertical (' $y$ ') deflections, which correspond to shifts perpendicular to and along the nozzlecatcher axis, respectively. The target thicknesses inferred from the measured yields at various deflections are shown in Figure 2. The properties of Gaussian fits to the measured yield profiles are given in Table 1.

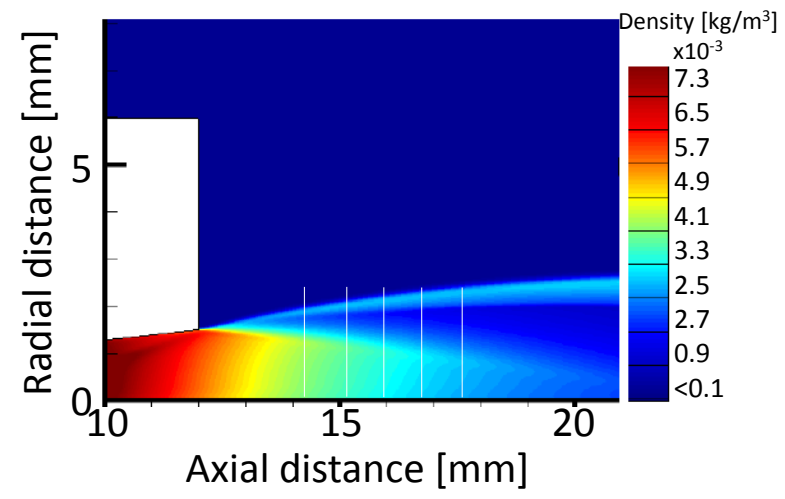

Figure 3: (color online.) Cross-sectional volumetric mass density profile of the HIPPO gas-jet from an ANSYS Fluent simulation using a 1 bar inlet pressure and $1.5 \times 10^{-3}$ bar ambient background pressure. The white rectangle represents the nozzle and the white vertical lines indicate the axial location and radial extent of the yield measurements performed in Reference [5].

\section{Gas-jet fluid dynamics simulations}

The state-of-the art computational fluid dynamics (CFD) software ANSYS Fluent ${ }^{2}$ (version 14.5.7) was employed to model the gas-dynamic properties of the HIPPO gas-jet target. Rather than simulate the entire 'central chamber' region of Reference [5], the simulations modeled the gasjet within the nozzle and expanding into an open volume. The omission of the remaining details of the central chamber, including the upstream and downstream collimators and gas catcher (each pictured in Figure 1), are justified as these features only serve to modify the central chamber pressure, which is a pressure boundary condition in the simulations. A fine mesh was used in the simulations to adequately resolve the spatial properties of the jet. As an exploratory approach, a two-dimensional axisymmetric model was adopted in order to reduce computation time. Only one layer of a two-dimensional mesh with 81,300 cells was considered, where the majority of cells were concentrated near the central jet region. As viscous effects are expected to be negligible near the gas-jet nozzle exit $[8,9]$, the inviscid flow assumption was adopted in the simulations for the purposes of our exploratory study in order to reduce computation time.

\footnotetext{
${ }^{2}$ http://www.ansys.com/Products/Fluids/ANSYS-Fluent
} 
For all simulations a helium jet was modeled using a total pressure of 1 bar at the nozzle inlet, a $3 \mathrm{~mm}$ nozzle-exit diameter, and a $9 \mathrm{~mm}$ neck-to-exit length (See Figure 2 of Reference [5].). The nozzle-neck diameter was chosen to be either $1 \mathrm{~mm}$, the nominal nozzle-neck diameter in Reference [5], or $0.72 \mathrm{~mm}$ with an ambient background pressure in the central chamber of either 1.5 mbar or 4.5 mbar to explore the sensitivity of the jet properties to the experimental design parameters. We were unable to use the $\sim 0.1$ mbar background pressures reported in Reference [5] and obtain numerical convergence due to the rapid dissipation of jet energy at the first downstream (i.e. increasing in axial distance from the nozzle) shock [10]. The impact of the increased background pressure is discussed in Section 5. Heat deposition from the ${ }^{20} \mathrm{Ne}$ ion beam in the gas-jet was not included, since past studies have shown it will not affect the jet properties [11].

The simulated volumetric mass density distribution for the gas-jet in the region near the nozzle exit to the location of the gas-catcher is shown for a $1 \mathrm{~mm}$ nozzle-neck diameter and 1.5 mbar background pressure in Figure 3. As expected for a supersonic expansion of a gas from a high-pressure nozzle into a low-pressure environment [12], the density rapidly declines along the nozzle-catcher axis with increasing axial separation from the nozzle's narrowest point. Contrary to suggestions by Reference [7] that the volumetric density $\rho$ along the axial direction $y$ should fall as $\rho \sim 1 / \sqrt{y}$ following the convergent-divergent nozzle exit, our simulation results favor the $\rho \sim 1 / y^{2}$ behavior they suggest should be more appropriate inside the nozzle. This is potentially due to the extra dimension in which gas can expand in our two-dimensional simulations, as opposed to their [7] one-dimensional approximation.

The other striking feature of Figure 3 is the brief, sharp rise in volumetric density at the outer radial extent of the gas-jet. This spike in volumetric density is due to the formation of a shock front by a Prandtl-Meyer expansion fan emanating from the convex corner at the nozzle neck and reflecting off of the jet boundary, resulting in compression waves [13-16]. This feature, which is generic to supersonic jets emerging from a convergent-divergent nozzle due to the strong shear between the supersonic jet and the rest gas, has been imaged in similar past studies through electron-beam induced fluorescence [14, 17] and Schlieren photography [13]. We find that increasing the background pressure within the central chamber enhances the volumetric density increase near the jet boundary and moves the jet boundary to narrower radii, as shown in Figures 4 and 5. Reference [13] ascribes the weakening of the volumetric density enhancement for a decrease in the background pressure with respect to the nozzle-exit pressure to an increase in turbulence for these conditions and therefore a smearing of the boundary where the jet and ambient gas mix.

The near-constant volumetric density region of the jet at inner radii is known as the 'potential core' [16]. As expected, the potential core becomes narrower with increas-

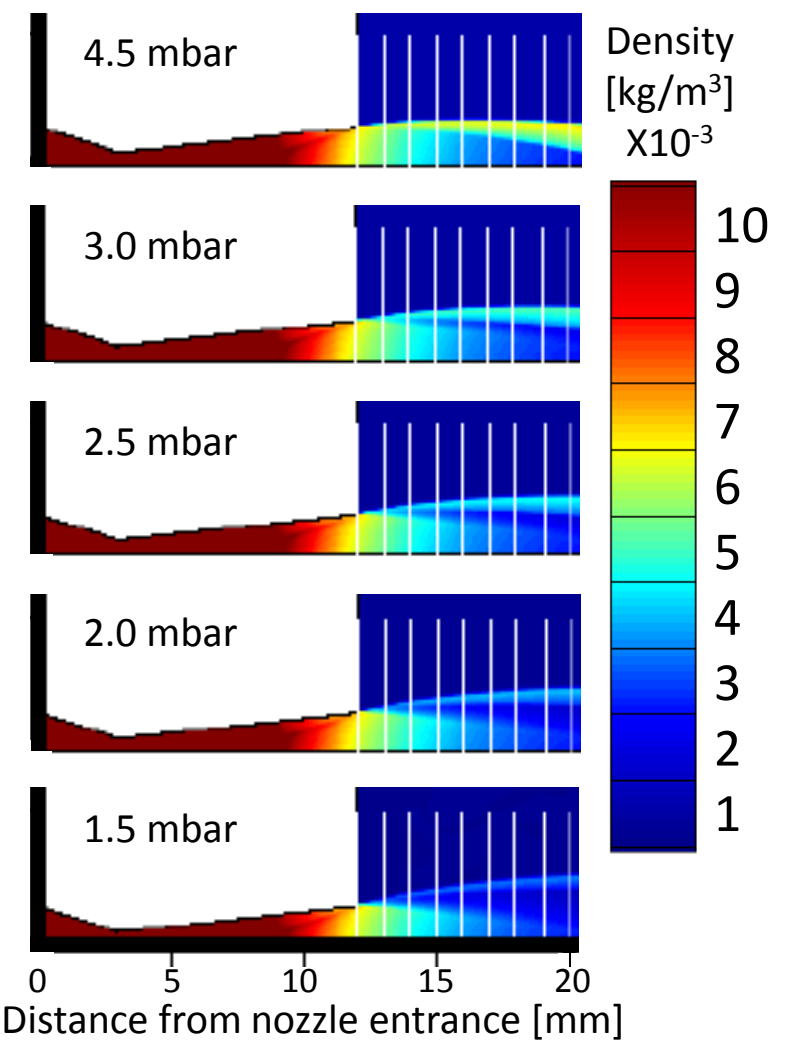

Figure 4: (color online.) Cross-sectional volumetric mass density profiles of the HIPPO gas-jet from ANSYS Fluent simulations using a 1 bar nozzle-inlet total pressure for five different background pressures. The region shown includes the nozzle to the axial distance where the HIPPO gas-catcher would be located out to a radial distance of $6 \mathrm{~mm}$. The white vertical lines, included for reference, are separated by $1 \mathrm{~mm}$.

ing axial distance from the nozzle, as seen in Figure 5.

The radial narrowing of the jet for higher background pressures (lower nozzle-exit to background pressure ratios) can be understood by considering the definition of the jet boundary as the location where the pressure of gas in the jet equals the ambient gas pressure, as is described in Reference [18]. As the gas in the jet moves further from the nozzle it initially expands in area (normal to the nozzlecatcher axis) and experiences a corresponding decrease in pressure. Since the jet-boundary is defined as a location of constant pressure, the decrease in pressure at the boundary of the jet due to the increase in jet area cannot drive the jet pressure below the pressure of the ambient background. Therefore, the jet boundary must turn inward toward the nozzle-catcher axis by some incremental angle while progressing from the nozzle to the first downstream (with respect to the nozzle exit) shock ('Mach disk'), which is located at axial distances larger than those shown in Figures 3 and 4 . For higher background pressures, the pressure of the jet is driven down to the ambient pressure by the increase in jet area at closer axial distances to the noz- 


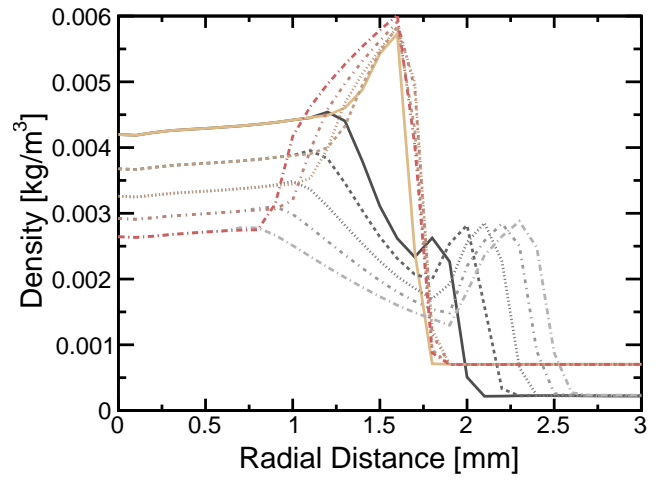

Figure 5: (color online.) Radial volumetric density profiles of the HIPPO gas-jet from ANSYS Fluent simulations at axial distances from the nozzle exit of $2,3,4,5$, and $6 \mathrm{~mm}$, in order of decreasing central density for a $1 \mathrm{~mm}$ nozzle-neck diameter, using background pressures of 1.5 mbar (gray lines) and 4.5 mbar (colored lines, sharing a maximum density at a radial offset of $\sim 1.6 \mathrm{~mm}$ ). The increased background pressure confines the jet to a narrower region, enhancing the high-density feature near the jet boundary. Note that the results were not normalized for mass-flow.

zle. Thus, for relatively higher background pressures, the supersonic gas-jet is narrower, as seen in Figures 4 and 5 . For the lower ambient pressure case, the jet-boundary is still expanding to larger radii at the axial distances from the nozzle which are shown in Figure 5, whereas the higher background pressure case reaches its apex in jet-boundary radius near this axial distance. Thus, the location of the jet boundary is roughly constant for the 4.5 mbar ambient pressure case.

For a fixed mass-flow, reducing the nozzle-neck diameter has the somewhat trivial impact of reducing the overall jet density. However, this would be recovered in the experimental set-up by increasing the gas charge in the system. The reduced jet-density, and therefore reduced ratio between the jet and ambient pressures, causes a narrowing of the jet, which is counteracted by the increased opening angle of the nozzle [13]. The combined impact on the jet aerial density profile is shown in Figure 6.

\section{Comparison between data and simulations}

In order to obtain a detailed comparison between the ANSYS Fluent simulation results and experimental data, we convert the simulated volumetric mass density distributions into distributions of aerial number density $N_{\mathrm{t}}$.

This is accomplished by evaluating the integral

$$
N_{\mathrm{t}, y}=\int n_{\mathrm{t}, y}(\ell) d \ell
$$

along a path through the jet $\ell$, where the volumetric number density distribution $n_{\mathrm{t}, y}(\ell)$ (atoms $/ \mathrm{cm}^{3}$ ) from the simulation results for a given axial offset $y$ is used. Since the nozzle is axisymmetric, so too is the jet structure and therefore our jet density distribution is described by a radial function of the volumetric mass density $\rho_{y}(r)$. When integrating along the longitudinal direction $x$, in which the ion beam traverses the gas-jet, a polar coordinate transformation is used to obtain a radial distance $r$ from the nozzle-catcher axis and therefore a volumetric density at that location. When integrating the jet thickness, locations with pressure below the ambient background pressure are ignored. This is because the ambient pressure in the experimental conditions is at least ten times lower the possible background pressures employed in the simulations, due to numerical convergence issues for too large a pressure range within a given simulation. Therefore, scattering of the ion beam off of ambient gas in the thickness measurement should have been negligible. The volumetric number density distribution $n_{\mathrm{t}}(x, y)$ (where $x$ and $y$ are used due to the Cartesian nature of the simulation grid) is trivially obtained from the simulation volumetric mass density distribution via the relationship $\rho=\frac{n_{t} m_{\text {mol }}}{N_{\mathrm{A}}}$, where $m_{\mathrm{mol}}$ is the molar mass of helium and $N_{\mathrm{A}}$ is Avogadro's number.

An additional correction must be made to account for the finite size of the ion beam as it impinges on the gas-jet. To account for this, we calculate an effective target thickness at each point $N_{\mathrm{t}}^{\mathrm{eff}}\left(x^{\prime}, y^{\prime}\right)$ by assuming a beam distribution of width $\Delta_{x}$ and weighting the local target thickness by the neighboring target thicknesses $N_{\mathrm{t}}(x+\delta x, y)$, where the weight $w(x+\delta x)$ is determined by the beam distribution shape. The resultant effective target thickness is

$$
N_{\mathrm{t}}^{\mathrm{eff}}\left(x^{\prime}, y^{\prime}\right)=\int_{x^{\prime}-\Delta_{x} / 2}^{x^{\prime}+\Delta_{x} / 2} w(x) N_{\mathrm{t}}\left(x, y^{\prime}\right) d x .
$$

Measurements reported in Reference [5] determined the beam width was $0.8 \mathrm{~mm}$, however, they neglected to note the beam distribution shape. Therefore, we have separately employed a uniform weighting distribution $0.8 \mathrm{~mm}$ wide and a Gaussian weighting distribution with a fullwidth at half-maximum of $0.8 \mathrm{~mm}$. As shown in Figure 6, we found little sensitivity to our choice of weight for the beam distribution. We do not perform a weighting over the axial dimension $y$, since, as seen in Figure 5, the increase and decrease in jet volumetric density for distances closer to and further from the nozzle exit are nearly equivalent and therefore essentially counteract each other. This was confirmed with a simple step-function weighting for a low resolution of axial distances.

The effective target thickness distributions derived from our ANSYS Fluent simulation results are compared to experimental data (shown separately in Figure 2) in Figure 6. The simulation results shown are for the axial distance at the nearest whole millimeter to the experimental results and assume two different nozzle-neck diameters and two different ambient background pressures. The simulation results have been scaled for three representative simulations which we have chosen to highlight, with three different scaling factors to roughly reproduce the measured 

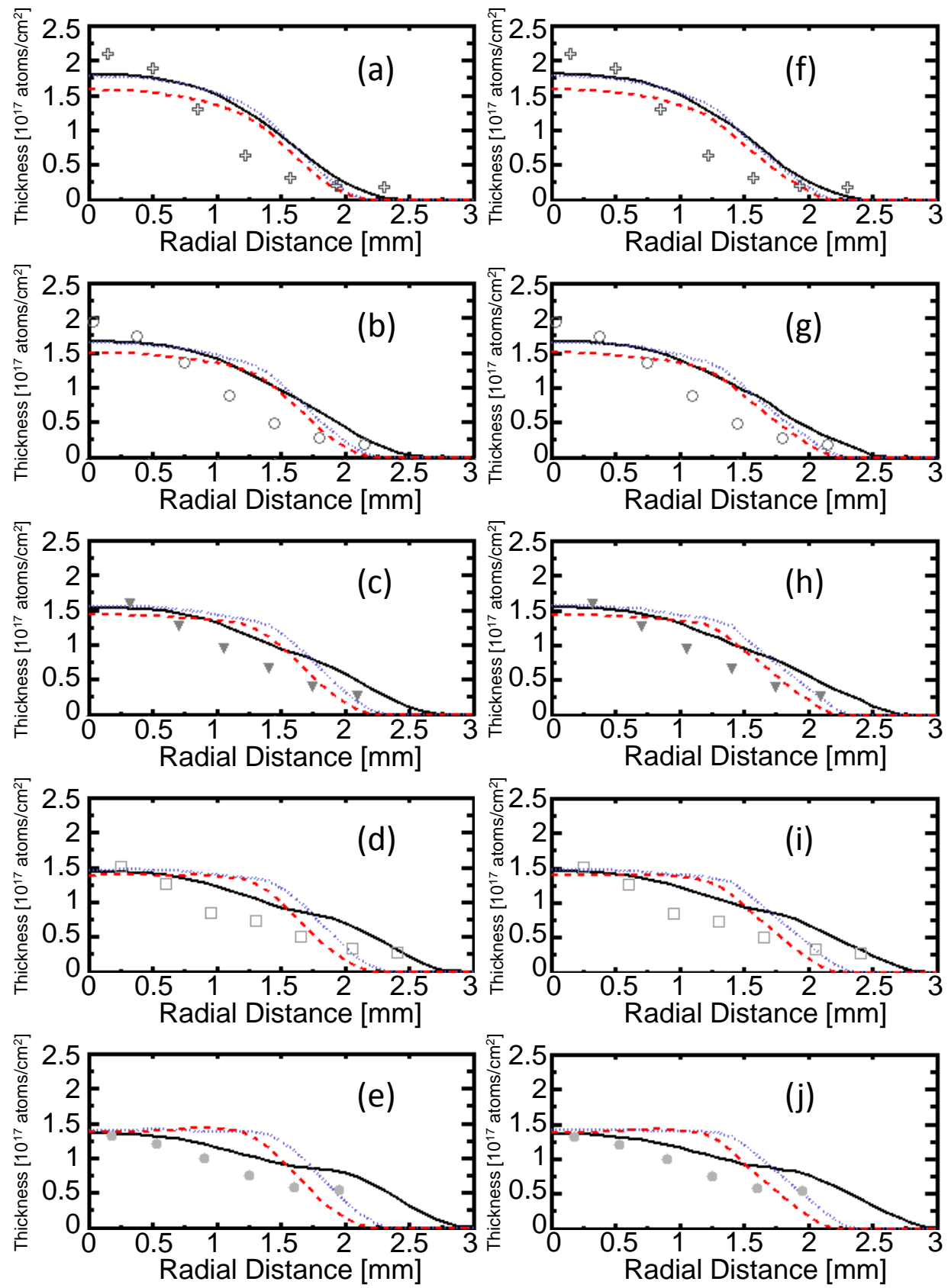

Figure 6: (color online.) Comparison between the measured (points) and simulated (lines) aerial density ('thickness') for a scan over the radial profile of the gas-jet at axial distances of $\sim 2-6 \mathrm{~mm}$ (top to bottom in $\sim 1 \mathrm{~mm}$ increments) from the nozzle exit. The simulation results in the left column assume a Gaussian distribution of the ion beam used for the elastic scattering measurement with a full-width at half-maximum of $0.8 \mathrm{~mm}$, while the simulation results in the right column assume a uniform ion beam distribution with a width of $0.8 \mathrm{~mm}$. The simulation conditions for the solid black, dashed red, and dotted blue curves are $d_{\text {nozzle-neck }}=1 \mathrm{~mm} p_{\text {background }}=1.5 \mathrm{mbar}, d_{\text {nozzle }- \text { neck }}=1$ mm $p_{\text {background }}=4.5 \mathrm{mbar}$, and $d_{\text {nozzle-neck }}=0.72 \mathrm{~mm} p_{\text {background }}=1.5 \mathrm{mbar}$, respectively. The magnitude of the three simulation results have been separately scaled $(\times 0.77,0.66$, and 1.5 for the black, red, and blue curves, respectively) to roughly match the measured thickness at the nozzle-catcher axis. Uncertainties for experimental data are obscured by the size of the data points. 
central target thickness. The unscaled simulation results reproduced the measured central thickness within $50 \%$.

\section{Discussion}

The radial target thickness profiles for various axial distances from the nozzle show qualitative agreement with the data (See Figure 6.) for each of the assumed ion beam distribution shapes and experiment design parameters. While the overall width of the gas-jet is reproduced, we find a more plateau-like radial behavior, resulting in a systematic overestimation of jet thickness near the half-width of the jet. This effect is less pronounced for simulations employing the nominal experimental nozzle-neck diameter and lowest ambient background pressure. Therefore, we expect the discrepancy at the half-width of the jet can partially be explained by our use of ambient background pressures that exceeded the experimentally measured value, which was required to achieve numerical convergence (See Section 3.). We surmise that increased mesh resolution around the regions containing large pressure gradients may remedy our numerical convergence issue, at the cost of computation time.

The remaining discrepancy between the data and simulation results is likely due to the two-dimensional treatment of a three-dimensional phenomenon. Employing twodimensions enforces an artificial symmetry that results in a systematic deviation of the simulated jet behavior from reality, for instance, shifting the location of the Mach disk (beyond the axial distances pictured in Figures 3 and 4) for all nozzle-exit/ambient pressure ratios [19, 20]. The deviation between simulated and measured jet densities is particularly severe for increased nozzle-exit/ambient pressure ratios [10]. It is interesting to note that hydrodynamic simulations of core collapse supernovae also have markedly different results for $2 \mathrm{D}$ and $3 \mathrm{D}$ simulations, in that case due to the inverse behavior of energy cascading between different length scales via turbulence [21]. In the present case, turbulent behavior within the nozzle may not be captured properly since our assumption of inviscid flow begins to break down for the relatively small Reynolds number corresponding to a millimeter-size nozzle with supersonic flow [22].

As seen in Figure 5, the qualitative impact of reducing the ambient background pressure is a broadening of the gas-jet and a lessening of the sharp volumetric density increase present near the jet boundary. These effects conspire to erase the plateau-like nature of the jet-thickness profile, resulting in a smoother transition from the central thickness to the ambient background conditions. Interestingly, modifying the neck-nozzle to a more narrow diameter while keeping the low ambient background pressure reinstates the plateau-like feature.

A comparison between the left and right columns of Figure 6 demonstrates an encouraging lack of sensitivity to our assumptions about the spatial distribution of the ion beam used to perform the thickness measurement of the jet via elastic scattering. We therefore conclude that our gas-jet behavior is primarily sensitive to design parameters of the gas-jet target system, in particular, the features impacting the ambient pressure of the target chamber. Nonetheless, future studies of gas-jet properties via elastic scattering would benefit from a measurement of the ion beam profile properties, beyond only the 'width'.

Contrary to prior expectations [5], we find the expected radial volumetric density distribution for the HIPPO gasjet differs markedly from the profile naïvely deduced from the elastic scattering yield data. We find the jet-density distribution does not resemble a Gaussian distribution. To the contrary, the volumetric density is rather constant near the nozzle-catcher axis and, following a period of decreasing density, increases again near the jet boundary. For relatively higher ambient pressures, the initial decrease in volumetric density in the radial direction is absent altogether. The substantial change in the jet-density distribution for increased background pressures is an important finding, as the introduction of a high atomic number gas into the central chamber is being considered to ensure charge-state equilibration of recoil ions exiting the gas-target, as has been done previously [23].

The enhancement in volumetric density near the jet boundary for increased background pressures may explain the reduced gas-catching efficiency observed by Reference [5] when argon is introduced into the HIPPO central chamber, since a larger fraction of the jet gas would flow towards the outer edge of the catcher (See Figure 4.). Counterarguments to this observation are that the catcher radius used in Reference [5] is larger than the radial extent of all jets simulated in this work and that, rather than narrow as expected, their measured jet distribution slightly broadened. We note that their result is somewhat counterintuitive since, as discussed in Section 3, arguments from first principles suggest that increased ambient pressures should result in a narrower jet boundary [18]. It is interesting to note that few numerical or experimental studies have been performed to date in order to determine the diameter of an underexpanded gas jet [16].

The case could be made that the details of the volumetric density distribution $n_{\mathrm{t}}(\ell)$ of the gas-jet are inconsequential, as it is the aerial number density ('target thickness') $N_{\mathrm{t}}$ that matters for a nuclear reaction cross section measurement [24]. However, the radial (with respect to the nozzle-catcher axis) density distribution of the gas-jet will be important to consider when optimizing HIPPO as a target for the St. George recoil separator. Due to the ion-optical design of St. George, the ability of the separator to accept recoil nuclei emitted from nuclear reactions is compromised by shifts of the target location from the assumed target region [6], as illustrated in Figure 1. If a substantial fraction of recoil nuclei were emitted from non-optimal positions, as would be the case if the target gas was primarily located at large radii from the nozzlecatcher axis, then a drastic underestimation of the deduced nuclear reaction cross section could occur. Furthermore, 
an extended target depth increases the effective recoil spot size at the target location, since the trajectories of ion optical rays are determined by the planar position and angle at the target center. This effect increases the width of recoils at the St. George mass-rejection slits, adversely affecting the beam-rejection [6].

Future studies will be critical to optimizing the performance of HIPPO as a nuclear reaction target for the St. George recoil separator. The experimental design parameters, e.g. nozzle properties, should be explored in a systematic study with ANSYS Fluent, including exploration of nozzles other than the convergent-divergent type and non-axisymmetric designs. This rapid prototyping can then be accompanied by the more time-consuming experimental verification studies of the gas-jet density distributions resulting from the most promising simulations. Given the ambiguity demonstrated in this work of elastic scattering yield data with respect to determining the gas-jet volumetric density profile, future verification studies should include alternative methods such as electronbeam induced fluorescence and Schlieren photography, if possible $[7,25]$. The findings of this and future studies of the fluid dynamic properties of the HIPPO gas-jet target will provide important input to help improve the performance of future gas-jet nuclear reaction targets required for recoil separators [26], reaction studies in ion storage rings [27, 28], and reaction studies requiring high beamintensities [25], which will play a pivotal role in advancing experimental nuclear astrophysics.

\section{Conclusions}

In summary, we have performed state-of-the-art CFD simulations with the software ANSYS Fluent of the HIPPO gas-jet target and compared our results to ${ }^{20} \mathrm{Ne}(\alpha, \alpha){ }^{20} \mathrm{Ne}$ elastic scattering measurements of the target thickness. We find qualitative agreement between our simulation results and experimental data. Our results demonstrate a strong sensitivity to the design conditions of the gas-jet target, highlighting the need for a systematic exploration of modifications of the nominal design in order to improve the performance of HIPPO as a nuclear reaction target. However, we note that the strength of our conclusions is limited by approximations presently employed in our simulations. Specifically, future CFD studies should explore the impact of moving from $2 \mathrm{D}$ to $3 \mathrm{D}$ and including viscous flow. More robust comparisons between simulations and data may be obtained by using redundant, alternative mechanisms for probing the jet volumetric density distribution, such as electron-beam induced fluorescence and Schlieren photography. The results obtained in this exploratory investigation are a crucial first step towards optimizing HIPPO as a nuclear reaction target for the St. George recoil separator. Future studies of the HIPPO jet properties will not only benefit the performance of HIPPO, but also gas-jet targets employed around the world for future nuclear reaction studies.

\section{Acknowledgements}

This material is based upon work supported by the National Science Foundation under Grants No. 1419765 and 1430152. We thank the University of Notre Dame Turbomachinery Laboratory for their support.

\section{References}

[1] G. Magkotsios, F. X. Timmes, A. L. Hungerford, C. L. Fryer, P. A. Young, M. Wiescher, The Astrophysical Journal Supplement Series 191 (2010) 66.

[2] M. Wiescher, F. Käppeler, K. Langanke, Annual Reviews in Astronomy and Astrophysics 50 (2012) 165.

[3] A. Caciolli, et al., The European Physical Journal A 39 (2009) 179.

[4] C. Ruiz, U. Greife, U. Hager, The European Physical Journal A 50 (2014).

[5] A. Kontos, D. Schürmann, C. Akers, M. Couder, J. Görres, D. Robertson, E. Stech, R. Talwar, M. Wiescher, Nuclear Instruments and Methods in Physics Research Section A 664 (2012) $272-281$.

[6] M. Couder, G. P. A. Berg, J. Görres, P. J. LeBlanc, L. O. Lamm, E. Stech, M. Wiescher, J. Hinnefeld, Nuclear Instruments and Methods in Physics Research Section A 587 (2008) 35 - 45.

[7] H. W. Becker, L. Buchmann, J. Görres, K. U. Kettner, H. Krüwinkel, C. Rolfs, P. Schmalbrock, H. P. Trautvetter, A. Vlieks, Nuclear Instruments and Methods in Physics Research 198 (1982) $277-292$.

[8] M. Pindzola, Jet Simulation in Ground Test Facilities, AGARDograph 79, North Atlantic Treaty Organization, Advisory Group for Aeronautical Research and Development, 1963.

[9] M. D. Salas, Proceedings of the American Institute of Aeronautics and Astronautics 7th Fluid and Plasma Dynamics Conference (1974) 523.

[10] R. Garcia, CFD simulation of flow fields associated with high speed jet impingement on deflectors, Master's thesis, Virginia Polytechnic Institute and State University, 2007. https://vtechworks.lib.vt.edu/handle/10919/31675.

[11] J. Görres, H. W. Becker, A. Krauss, A. Redder, C. Rolfs, H. P. Trautvetter, Nuclear Instruments and Methods in Physics Research Section A 241 (1985) $334-338$.

[12] J. Ulbricht, G. Glausnitzer, G. Graw, Nuclear Instruments and Methods in Physics Research Section A 102 (1972) 93-99.

[13] E. K. Latvala, T. P. Anderson, Planetary and Space Science 4 (1961) 77-91.

[14] R. Herron, Investigation of jet boundary simulation parameters for underexpanded jets in a quiescent atmosphere, Technical Report AEDC-TR-68-108, Arnold Engineering Development Center, 1968.

[15] T. S. Cheng, K. S. Lee, International Journal of Heat and Fluid Flow 26 (2005) 755-770.

[16] E. Franquet, V. Perrier, S. Gibout, P. Bruel, Progress in Aerospace Sciences 77 (2015) 25-53.

[17] M. Belan, S. De Ponte, D. Tordella, Physical Review E 82 (2010) 026303.

[18] T. C. Adamson, Jr., J. A. Nicholls, Journal of the Aerospace Sciences 26 (1959) 16-24.

[19] J. Wilkes, C. Glass, P. Danehy, R. Nowak, Proceedings of the 44th AIAA Aerospace Sciences Meeting and Exhibit (2006) 910.

[20] M. Cinalli, R. Keppens, Journal of Physics D 39 (2006) 4589.

[21] H.-T. Janka, T. Melson, A. Summa, Annual Reviews of Nuclear and Particle Science 66 (2016) 1-35.

[22] W. F. Louisos, D. L. Hitt, Journal of Spacecraft and Rockets 51 (2014) 491

[23] D. Schürmann, et al., Nuclear Instruments and Methods in Physics Research A 531 (2004) 428-434.

[24] C. E. Rolfs, W. S. Rodney, Cauldrons in the Cosmos: Nuclear Astrophysics, University of Chicago Press, 1988. 
[25] F. Favela, et al., Physical Review Accelerators and Beams 18 (2015) 123502.

[26] K. Chipps, et al., Nuclear Instruments and Methods in Physics Research A 763 (2014) 553 - 564.

[27] M. Grieser, et al., The European Physical Journal Special Topics 207 (2012) 1-117.

[28] B. Mei, et al., Physical Review C 92 (2015) 035803. 